\title{
Correction to: Analysis of FOXO3 Gene Polymorphisms Associated with Human Longevity
}

\section{Timothy A. Donlon, Philip M. C. Davy, and Bradley J. Willcox}

Correction to:

Chapter 21 in: Wolfgang Link (ed.), FOXO Transcription Factors:

Methods and Protocols, Methods in Molecular Biology, vol. 1890, https://doi.org/10.1007/978-1-4939-8900-3_21

The original version of this chapter was inadvertently published with only one affiliation for Philip M. C. Davy. Additional affiliation has now been included in chapter metadata and front matter

Philip M. C. Davy

Institute for Biogenesis Research, University of Hawaii, Honolulu, HI, USA

Department of Research, Kuakini Center for Biomedical Research Excellence (COBRE), Kuakini Medical Center, Honolulu, HI, USA

This chapter was partially supported by the COBRE - Center for Translational Research on Aging at Kuakini Medical Center, with a grants from the National Institute of General Medical Sciences - NIGMS (1 P20 GM125526-01) from the National Institutes of Health. Unfortunately, these grants were not acknowledged in the original version.

The updated online version of this chapter can be found at: https://doi.org/10.1007/978-1-4939-8900-3_21 